\title{
Solitons in semiconductor microcavities
}

To the Editor - In a recent Article published in Nature Photonics ${ }^{1}$, Sich et al. reported the observation of bright polariton solitons in a semiconductor microcavity. As part of their analysis, the authors discussed the response time of polariton solitons in comparison with the response time of what they called "lightonly solitons in semiconductor cavity lasers" or "pure-light cavity solitons in wide-aperture semiconductor lasers", citing work of Barland et al. ${ }^{2}$, Pedaci et al. ${ }^{3}$ and Ackemann et al. ${ }^{4}$

In this correspondence, we want to remark that the cavity solitons studied by Barland et al. ${ }^{2}$ and Pedaci et al. ${ }^{3}$, and reviewed by Ackemann et al. ${ }^{4}$, are actually composite structures consisting intrinsically of both a light and a material component, and that referring to them as 'pure light' is therefore not appropriate.

Indeed, there is of course no nonlinear optics without a medium and any modulation of the light field will have a counterpart in terms of material variables, be it electronic states, Zeeman states, twist angles of liquid-crystal directors, carrier populations or coherences. Depending on the relative timescales involved, the material dynamics might be adiabatically eliminated, making this connection less obvious but not less real.

In the specific case of semiconductor microcavities referred to by Sich et al. ${ }^{1}$, the relevance of the material component has been envisaged since the very first studies of cavity solitons in semiconductor systems $^{2,5}$, and the impact of material timescales on cavity solitons nucleation and motion has actually been discussed by Pedaci et al. ${ }^{3}$ and Ackemann et al. ${ }^{4}$, among others. For instance, dedicated numerical analyses ${ }^{6}$ have shown that forcing a spatial separation of the material and optical components of a cavity soliton causes it to disappear, further demonstrating the intrinsic 'light-matter' nature of cavity solitons in semiconductor microcavity models. Perhaps even more significantly, this composite nature has been exploited in experiments that involve controlling cavity solitons by optically manipulating their material component ${ }^{7}$.

In view of these observations, we believe that the terms 'light only' and 'pure-light' do not accurately reflect the nature of semiconductor cavity solitons and are therefore not helpful in framing a quantitative or qualitative comparison with polariton solitons.
References

1. Sich, M. et al. Nature Photon. 6, 50-55 (2012).

2. Barland, S. et al. Nature 419, 699-702 (2002).

3. Pedaci, F. et al. Appl. Phys. Lett. 92, 011101 (2008).

4. Ackemann, T., Firth, W. J. \& Oppo, G.-L. in Advances in Atomic Molecular and Optical Physics Vol. 57 (eds Berman, P. R., Arimondo, E. \& Lin, C. C.) Ch. 6, 323-421 (Academic, 2009).

5. Brambilla, M., Lugiato, L. A., Prati, F., Spinelli, L. \& Firth, W. J. Phys. Rev. Lett. 79, 2042-2045 (1997).

6. Prati, F., Tissoni, G., McIntyre, C. \& Oppo, G.-L. Eur. Phy. J. D 59, 139-147 (2010).

7. Barbay, S. et al. Opt. Lett. 31, 1504-1506 (2006).

S. Barland ${ }^{1 \star}$, M. Giudici', G. Tissoni', J. R. Tredicce', M. Brambilla' ${ }^{2}$ L. Lugiato ${ }^{3}$, F. Prati $^{3}$, S. Barbay ${ }^{4}$, R. Kuszelewicz ${ }^{4}$, T. Ackemann ${ }^{5}$, W. J. Firth ${ }^{5}$ and G.-L. Oppo ${ }^{5}$

${ }^{1}$ Institut Non Linéaire de Nice, Université de Nice Sophia Antipolis - CNRS UMR

7335, 1361 Route des Lucioles, 06560

Valbonne, France. ${ }^{2}$ CNISM, CNR-LIT3

and Dipartimento di Fisica Interateneo

Politecnico e Università di Bari, Via

Amendola 173, Bari 70126, Italy. ${ }^{3}$ Università

dell'Insubria, Dipartimento di Scienza

e Alta Tecnologia, via Valleggio 11,

22100 Como, Italy. ${ }^{4}$ Laboratoire de

Photonique et de Nanostructures -

CNRS UPR20, Route de Nozay, 91460

Marcoussis, France. ${ }^{5}$ SUPA and the Department of Physics, University of Strathclyde,

Glasgow G4 ONG, Scotland, UK.

*e-mail: stephane.barland@inln.cnrs.fr
Sich et al. reply: Regarding the terminology used in our recent publication (Sich, M. et al. Nature Photon. 6, 50-55; 2012), we want to point out that it is known that both the linear and nonlinear parts of the refractive index contribute to soliton formation and are intrinsic optical properties of matter. Understanding the impact of these properties on the propagation of photons, such as dispersion, nonlinear frequency conversion and soliton formation, does not in most cases require a departure from the concept of photons. This is the limit of the 'weak coupling' between light and matter.

The first example of solitons we gave in the introduction of our work is that of solitons in optical fibres, which are pulses of light and are thus readily described in the limit of weak light-matter interaction. We therefore referred to these as 'light-only solitons'. In the case of strong coupling between light and matter, as is realized in the semiconductor microcavity used in our work, we deal with excitation frequencies where the cavity (and therefore light) resonance is absent and the excitonic resonance is also absent, so that neither light nor excitonic (matter) waves exist for the frequencies under consideration. Only half-light, half-matter polaritonic resonance exists at these frequencies, and we therefore refer to these as half-light, half-matter solitons - or 'polariton solitons'.

Investigations of microcavity solitons cited in the correspondence by Barland et al. deal with the case of weak coupling, where the energy levels of photons and material excitations do not hybridize and no polaritonic quasiparticles are formed. As such, in our terminology, this falls into the category of light-only solitons, which, as in optical fibres and other settings, cannot exist without the surrounding matter. In this respect we should also mention that adiabatic or non-adiabatic dynamics of the material excitations is a factor that is secondary to the existence (or non-existence) of polaritonic energy levels and to the choice of operating frequency with respect to these levels. We hope our comments here address the terminology concerns discussed in the correspondence by Barland et al.

D. V. Skryabin ${ }^{1 \star}$, D. N. Krizhanovskii², M. S. Skolnick ${ }^{2}$ E. A. Cerda-Méndez ${ }^{3}$ and R. Hartley'

'Department of Physics, University of Bath, Bath BA2 7AY, UK. ²Department of Physics and Astronomy, University of Sheffield, Sheffield S3 7RH, UK. ${ }^{3}$ Paul-Drude-Institut für Festkörperelektronik, Berlin, Germany.

*e-mail: d.v.skryabin@bath.ac.uk 